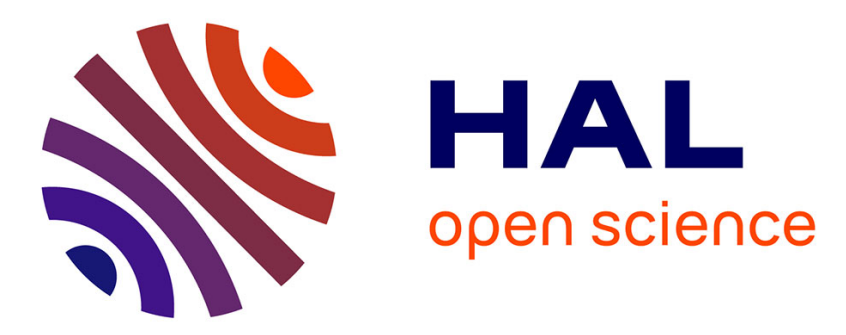

\title{
Local stability of hydrostatic compression states of non-linearly thermo-visco-elastic bodies of differential type
}

Victor A. Eremeyev

\section{- To cite this version:}

Victor A. Eremeyev. Local stability of hydrostatic compression states of non-linearly thermo-viscoelastic bodies of differential type. Journal of Applied Mathematics and Mechanics, 1991, 55 (2), pp.259-265. hal-00830569

\section{HAL Id: hal-00830569 \\ https://hal.science/hal-00830569}

Submitted on 5 Jun 2013

HAL is a multi-disciplinary open access archive for the deposit and dissemination of scientific research documents, whether they are published or not. The documents may come from teaching and research institutions in France or abroad, or from public or private research centers.
L'archive ouverte pluridisciplinaire HAL, est destinée au dépôt et à la diffusion de documents scientifiques de niveau recherche, publiés ou non, émanant des établissements d'enseignement et de recherche français ou étrangers, des laboratoires publics ou privés. 


\section{LOCAL STABILITY OF HYDROSTATIC COMPRESSION STATES OF NON-LINEARLY THERMO-VISCO-ELASTIC BODIES OF DIFFERENTIAL TYPE*}

\section{V.A. EREMEYEV}

The local Lyapunov stability of a hydrostatically stressed state of an isotropic, homogeneous, non-linearly thermo-visco-elastic body of differential type with complexity $n$ and constant initial temperature is investigated in a dynamical formulation. The connection between the thermal and mechanical fields is taken into account. As a special case a model of an incompressible non-linearly visco-elastic body is investigated. Conditions that ensure the existence and uniqueness of generalized solutions of the linearized equations of motion and heat conduction and their vanishing with time are formulated. The example shows that violation of the conditions obtained can lead to the exponential growth of the solution.

In the investigation of the local stability of hydrostatically stressed states (HSSs) of non-1inearly elastic bodies by static methods /1, 2/ the absence of adjacent equilibrium forms came to the force as a necessary requirement placing restrictions on the form of the equation of state of the material. The stability of the solutions of the equilibrium equations of non-linear visco-elastic bodies with integral defining relations has been investigated /3-5/. The conditions for the stability of an HSS of a visco-elastic body were obtained in 14/. The influence of the temperature field was taken into account in /5/. On the basis of the theory developed in $/ 6 /$, the behaviour with time of solutions of the equations of motion of linear visco-elastic bodies of aifferential type has been investigated /7/.

1. The fundamental relations describing a homogeneous isotropic non-linearly thermovisco-elastic body of differential type with complexity $n$ have the form $/ 8 /$

$$
\begin{aligned}
& \Psi=\psi(\mathbf{G}, \theta), \eta=-\partial \psi / \partial \theta(\mathbf{G}, \boldsymbol{\theta}), \mathbf{T}=\mathbf{T}_{0}(\theta, \mathbf{F})+\mathbf{T}_{\mathbf{1}}\left(\theta, \mathbf{F}, \mathbf{A}_{1}, \ldots\right. \\
& \left.\mathbf{A}_{n}\right) \\
& \mathbf{T}_{0}=2 \rho \mathbf{C}^{\mathbf{T}} \cdot \partial \psi / \partial \mathbf{G} \cdot \mathbf{C}, \mathbf{h}=\mathbf{h}(\Theta, \nabla \Theta, \mathbf{F}), \mathbf{G}=\mathbf{C} \cdot \mathbf{C}^{\mathbf{T}}, \mathbf{F}=\mathbf{C}^{\mathbf{T}} \cdot \mathbf{C}
\end{aligned}
$$

Here $\psi$ and $\eta$ are the mass densities of the free energy and entropy, $T$ is the Cauchy stress tensor, $T_{0}$ and $T_{1}$ are the equilibrium and dissipative stress tensors, $h$ is the heat flux vector, $\theta$ is the temperature, $C$ is the deformation gradient, $G$ and $F$ are the CauchyGreen and Finger deformation measures, $\rho$ is the denisty of the body in the current configuration, $\nabla$ is the gradient operator in the current configuration, and the $\mathbf{A}_{k}(k=1, \ldots, n)$ are RivlinEricksen tensors, defined by the formula /9/

$$
\mathbf{A}_{k}=\mathbf{C}^{-1} \cdot\left(d^{k} \mathrm{G} / d t^{k}\right) \cdot \mathbf{C}^{-T}, k=1, \ldots, n
$$

Instead of the tensors $A_{k}$ one can use other indifferent tensors characterizing the deformation velocities in relations (1.1). The tensor $\mathbf{T}_{1}$ vanishes for $\mathbf{A}_{k}=0(k=1, \ldots, n)$. When considering a purely mechanical theory one uses a model of an incompressible viscoelastic material with defining relations for the Cauchy stress tensor of the form

$$
\mathbf{T}=-p \mathbf{E}+\mathbf{T}_{0}(\mathbf{F})+\mathbf{T}_{\mathbf{1}}\left(\mathbf{F}, \mathbf{A}_{1}, \ldots, \mathbf{A}_{n}\right)
$$

where $p$ is the unknown function determined from the equations of motion and the incompressibility condition $\operatorname{det} \mathbf{G}=1$, and $\mathbf{E}$ is the unit tensor.

An equilibrium state of the body which is in an HSS and has a uniform temperature field will be called a $\left(p, \theta^{\circ}\right)$-configuration. A $\left(p, \theta^{\circ}\right)$-configuration of an isotropic body satisfies the relations $T=-p E, \theta=\theta^{\circ}, \mathbf{C}=l \mathbf{Q}, \quad \mathbf{G}=\mathbf{F}=l^{2} \mathbf{E}, \mathbf{A}_{k}=0 \quad$ and $\quad \mathbf{h}=0$, where $\mathbf{Q}$ is a proper orthogonal tensor and $0<l \leqslant 1$. For an incompressible material $l=1$.

In order to investigate the stability of this state, the equations of motion and heat conduction are linearized about the $\left(p, \theta^{\circ}\right)$-configuration. In the metric of the deformed state the linearized equations have the form $/ 1,9 /$ 


$$
\begin{gathered}
F \cdot \mathbf{I}=\rho \partial_{t}^{2} \mathbf{w}, \quad \mathbf{I}=\delta \mathbf{T}+\mathbf{T} \operatorname{tr} \mathbf{L}-\mathbf{L}^{T} \cdot \mathbf{T}, \quad \mathbf{L}=\nabla \mathbf{w} \\
\delta \mathbf{T}=\frac{d}{d \xi} \mathbf{T}\left(\mathbf{R}+\xi \mathbf{w}, \Theta^{\circ}+\xi \theta\right)_{\xi=0,} \quad \partial_{t}=\frac{\partial}{\partial t}
\end{gathered}
$$

Here $\mathbf{R}$ is the radius-vector in the $\left(p, \theta^{\circ}\right)$-configuration, $\mathbf{w}$ is a small additive displacement vector field and $\theta$ is the temperature shift. The symbol $\delta$ denotes linear perturbations caused by the displacements and temperature shifts. we obtain

Linearizing the Rivlin-Ericksen tensors in the neighbourhood of the $(p$, $\theta$ ) configuration

$$
\delta \mathbf{A}_{k}=2 \partial_{t}{ }^{k} \boldsymbol{\varepsilon}, \quad 2 \boldsymbol{e}=\mathbf{L}+\mathbf{L}^{T}
$$

Following $/ 2 /$, we transform the equations of motion linearized in the neighbourhood of the $\left(p, \theta^{\circ}\right)$ configuration to the form

$$
\nabla \cdot S=\rho \partial_{t}^{2} \mathbf{w}, S=\delta \mathbf{T}-\mathbf{\Omega} \cdot \mathbf{T}+\mathbf{T} \cdot \mathbf{\Omega}, 2 \boldsymbol{\Omega}=\mathbf{L}^{T}-\mathbf{L}
$$

Taking account of relations $(1.1)$ and (1.5) and the isotropy conditions, one can show that $\mathrm{S}$ is a linear isotropic function of the tensors $\partial_{t}^{k} \varepsilon(k=0, \ldots, n)$ and $\theta$;

$$
\begin{gathered}
\mathrm{S}=\Lambda\left(\partial_{t}\right) \operatorname{tr} \mathbf{8} \mathbf{E}+2 M\left(\partial_{t}\right) \varepsilon-\alpha(l) \theta \mathbf{E} \\
\Lambda(\zeta)=\sum_{k=0}^{n} \lambda_{k}(l) s^{k}, M(s)=\sum_{k=0}^{n} \mu_{k}(l) s^{k}
\end{gathered}
$$

Relations (1.7) define the response of the material to small deformations near the HSS. For an incompressible body

$$
\mathbf{S}=-\delta p \mathbf{E}+2 M\left(\partial_{t}\right) \mathbf{e}
$$

where $\delta p$ is an unknown function of the coordinates, for which the linearized incompressibility condition $\nabla \cdot w=0$ serves as a supplementary defining condition.

To obtain linearized heat-conduction equations we consider the energy-balance equation in the case when there are no bulk heat sources /8-12/:

$$
\rho d(\psi+\theta \eta) / d t=-\nabla \cdot \mathbf{h}+\operatorname{tr}(\mathbf{T} \cdot \nabla d \mathbf{R} / d t)
$$

Using relation (1.1), this equation can be transformed to the form

$$
\rho \theta d \eta / d t=-\Gamma \cdot \mathbf{h}+\operatorname{tr}\left(\mathbf{T}_{\mathbf{1}} \cdot \nabla d \mathbf{R} / d t\right)
$$

The last term on the right-hand side of this equation describes the specific dissipation of energy $19,12 /$ for non-Iinear thermo-visco-elastic bodies of differential type. Ihe presence of the dissipative term distinguishes the energy-balance equation under consideration from the heat-conduction equation for non-linear thermo-elastic bodies. Taking account of relation (1.1) and the material isotropy condition, we write down expressions for the entropy and heat flux vector linearized in the neighbourhood of the $\left(p, \theta^{\circ}\right)$-configuration:

$$
\rho \delta \eta=c(l) \theta+\alpha(l) \nabla \cdot \mathbf{w}, \delta \mathbf{h}=-x(l) \theta \nabla
$$

In a small neighbourhood of the $\left(p, \Theta^{\circ}\right)$-configuration the dissipative term in the heatconduction equation, which vanishes in that configuration (because in equilibrium configurations, of which the $\left(p, \theta^{\circ}\right)$-configuration is a special case, $\mathbf{T}_{1}$ and $d \mathbf{R} / d t$ are both zero), has second order of smallness compared with the other terms and vanishes on 1 inearization. The last linearization is of the heat-conduction equation in a neighbourhood of the $\left(p\right.$, $\left.\theta^{\circ}\right)$ configuration, and has the form

$$
c(l) \Theta^{\circ} \partial_{t} \theta=x(l) \nabla \cdot \nabla \theta-\alpha(l) \Theta^{\circ} \partial_{t} \nabla \cdot w
$$

The derivation of the non-linear heat-conduction equation and its linearization about an unstressed state of visco-elastic bodies of differential type 1 was performed in /12/. The form of the linearized heat-conduction equation obtained in /12/ is similar to (1.9).

The quantities $c(l)$ and $x(l)$ will be regarded as positive. The initial temperature $\theta^{\circ}$ will without loss of generality be taken to be equal to unity. The linearized boundary conditions on the surface of the body $\Sigma=\Sigma_{1} \cup \Sigma_{2} \cup \Sigma_{3}=\Sigma_{4} U$ $\Sigma_{5}$ have the form $/ 2 /$

$$
\left.\mathbf{w}\right|_{\mathbf{x}_{1}}=0,\left.\mathbf{N} \cdot \mathbf{S}\right|_{\mathbf{s}_{2}}=0,\left.\mathbf{N} \cdot \mathbf{w}\right|_{\mathbf{s}_{\mathbf{t}}}=0
$$

$$
\left.\mathbf{N} \cdot \mathbf{S} \cdot(\mathbf{E}-\mathbf{N} \mathbf{N})\right|_{\Sigma_{s}}=0,\left.\theta\right|_{\Sigma_{*}}=0,\left.\mathbf{N} \cdot \nabla \theta\right|_{\Sigma_{s}}=0
$$

(where $\mathbf{N}$ is the normal to the surface of the body). The $\Sigma_{1}$ component of the surface of the body is completely fixed, a uniform hydrostatic pressure is assigned to the component $\Sigma_{2}$, on $\Sigma_{3}$ the body is in contact with a smooth solid surface, the temperature is specified on $\Sigma_{\text {, }}$ and the heat flux on $\Sigma_{5}$. 
The initial conditions for $w$ and $\theta$ are written as follows:

$$
\left.\partial_{t}{ }^{k} \mathbf{w}\right|_{t=0}=w_{k}, k=0, \ldots, N \equiv \max (1, n-1),\left.\theta\right|_{t=0}=\theta_{0}
$$

A solution of the resulting initial-boundary value problems will be sought in the form

$$
\mathbf{w}=\mathbf{u}+\chi(t) \sum_{k=0}^{N} \frac{t^{k}}{k !} \mathbf{w}_{k}, \quad 0=\tau+\chi(t) \theta_{0}
$$

where the infinitely differentiable function $\chi(t)=1$ in the neighbourhood of zero and $\chi(t)=$ 0 for $t>1$. The functions $u$ and $\tau$ satisfy homogeneous initial and boundary conditions and inhomogeneous equations of motion and heat conduction with some fictitious "body forces" f and "heat sources" $q$.

2. To investigate the resulting initial-boundary value problems we will use the Laplace transformation (with $s$ as the transform parameter).

As in /7/ we introduce the following spaces.

Suppose $H$ is some separable Hilbert space.

$E_{\mathrm{k}}(\gamma, H)$ (where $k$ is an integer and $\gamma \geqslant 0$ ) is the space of functions with values in $H$, analytic in the half-plane $\operatorname{Re} s>\gamma$ and with finite norm

$$
\|\varphi\|_{E_{k}}^{2}=\sup _{\sigma>\gamma} \int_{-\infty}^{+\infty}\|\varphi(\sigma+i \tau)\|_{H^{2}}\left(1+|\sigma+i \tau|^{2 k}\right) d \tau
$$

$P_{k}(\gamma, H)$ is a complex space of functions $g(t)$ with values in $H$, possessing generalized derivatives $/ 10 /$ on $[0,+\infty)$ up to order $k$ inclusive, such that $\partial_{t}{ }^{m} g=0 \quad$ for $t=0$ $(0 \leqslant m \leqslant k)$, and a finite norm

$$
\|g\|_{p_{k}}^{2}=\int_{0}^{+\infty} e^{-2 v t} \sum_{m=0}^{k}\left\|\partial_{t}^{m} g\right\|_{H}^{2} d t
$$

A theorem has been proved $/ 7 /$ to the effect that the Laplace transformation operator continuously maps the space $P_{k}(\gamma, H)$ (where $k \geqslant 0$ is an integer and $\gamma>0$ ) onto the space $E_{\mathrm{k}}(\gamma, H)$, and that it is continuously invertible and that its inverse serves as the inverse Laplace transform operator.

Below we shall need the following assertions.

Lenma 2.1. Suppose the roots of the polynomial $P(s)=p_{0}+p_{1} s+\ldots+p_{n} s^{n}$ lie in the left half-plane of the complex plane and $p_{n}>0$, then for $\operatorname{Re} s \geqslant 0$ we have the inequality $|P(s)| \geqslant d p_{n}\left(1+|s|^{2^{n}}\right)^{1 / 2}$, where $d \leqslant 1$.

The proof follows from expanding the polynomial $P(s)$ in simple factors and the incqualities $\left|s-s_{k}\right| \geqslant d\left(1+|s|^{2}\right)^{1 / 3}$ with $d<1$ for $\operatorname{Re} s_{k}<0$.

3. We will consider the first initial-boundary value problem for a compressible thermovisco-elastic body $(1.6)$, (1.7), (1.9), (1.10) (with $\Sigma=\Sigma_{1}=\Sigma_{4}$ ), which for brevity we shall call problem A.

Let $H_{1}$ be the space of complex-valued functions formed by the closure of the set of continuously differentiable functions equal to zero on the boundary $\omega$, in the norm

$$
\|\tau\|_{H_{1}}^{2}=\iiint \nabla \tau \cdot \nabla \tau d \omega
$$

Here and below we shall not specify the domain of integration of $\omega . H_{2}$ is the space of vector functions each of whose components belong to $H_{1}$. It is obvious that $H_{1}=W_{2}{ }^{0(1)}$ and $H_{2}=W_{2}{ }^{0(1)} W_{2}{ }^{0(1)} \times W_{2}{ }^{(1)}$.

Applying a Laplace transformation to problem A one obtains a boundary-value problem with a parameter - problem B. We shall call $v(s) \in H_{2}, \tau(s) \in H_{1}$ a generalized solution of problem B, if for arbitrary functions $g \in H_{2}$ and $\varphi \in H_{1}$ we have the equalities

$$
\begin{gathered}
\iiint\left\{M(s) \nabla v \cdots \nabla \bar{g}^{T}+[M(s)+\Lambda(s)] \nabla \cdot v \nabla \cdot \overline{\mathbf{g}}+\right. \\
\left.\rho s^{2} \mathrm{v} \cdot \overline{\mathbf{g}}-\alpha \tau \nabla \cdot \overline{\mathrm{g}}-\mathbf{F} \cdot \overline{\mathbf{g}}\right\} d \omega=0 \\
\iiint[x \nabla \tau \cdot \nabla \bar{\varphi}+c s \tau \bar{\varphi}-\alpha s \mathbf{v} \cdot \nabla \varphi-Q \bar{\varphi}] d \omega=0 \\
\left(\mathbf{F}=L \mathrm{f} \in E_{0}\left(0, H_{2}^{-1}\right), Q=L q \in E_{0}\left(0, H_{1}^{-1}\right)\right)
\end{gathered}
$$

We shall perform the proof of the existence of a generalized solution to problem $B$ in two stages. Regarding the vector $v$ as given, we will investigate the problem of finding the 
temperature, taking as the definition of the solution the satisfaction of relation (3.2) by any function $\varphi \in H_{1}$. Using the Riesz theorem, Eq. (3.2) can be represented in the form of an operator equation in the space $H_{1}$ :

$$
\begin{gathered}
A(s) \tau \equiv x \tau+c s K \tau=\Phi, \Phi=K Q+\alpha s K, \mathbf{v} \\
(K Q, \varphi)_{H_{s}}=\iiint Q \bar{\varphi} d \omega,\left(K_{1} \mathbf{v}, \varphi\right)_{H_{s}}=\iiint \mathrm{v} \cdot \nabla \bar{\varphi} d \omega
\end{gathered}
$$

The operator $K$ acts continuously from $H_{1}^{-1}$ into $H_{1}$ and the operator $K_{1}$ acts continuously from $L_{2}$ into $H_{1}$. One can show that $\left\|K_{1}\right\|=1$.

Lema 3.1. Suppose $\mathrm{v} \in E_{n}\left(0, L_{2}\right)$. Then Eq. (3.3) is uniquely soluble for Res $>0$ and its solution can be represented in the form

$$
\tau=\tau_{1}+\tau_{22} \tau_{1} \in E_{0}\left(0, H_{1}\right), \tau_{2} \in E_{n-1}\left(0, H_{1}\right) \cap E_{n}\left(0, L_{2}\right)
$$

Proof. We have the following equalities:

$$
\begin{gathered}
(A(s) \tau, \tau)_{H_{1}}=P_{0}(s)(\tau, \tau)_{H_{1}}=P_{1}(s)(\tau, \tau)_{L_{,}} \\
\left(P_{0}(s)=x+c k s, P_{1}(s)=c s+k^{-1} x, k=\|\tau\|_{L_{0}}^{2}\|\tau\|_{H_{1}}^{-2}\right.
\end{gathered}
$$

The parameter $k \in\left(0, k^{\circ}\right)$, where the quantity $k^{\circ}$ depends on the domain 0.

Using Lemma 2.1 , the inequality

$$
\begin{gathered}
\left|(A(s) \tau, \tau\rangle_{H_{1}}\right| \geqslant \zeta(s)\|\tau\|_{L_{*}}^{2}\left|(A(s) \tau, \tau)_{H_{1}}\right| \geqslant x\|\tau\|_{H_{L}}^{z} \\
\zeta(s)=\min \left(c, x / k^{\circ}\right)\left(1+|s|^{*}\right)^{1 / /}
\end{gathered}
$$

follows from the relations obtained when Res $>0$.

It follows from (3.4) that Eq. (3.3) is properly solvable and the operator $A(s) \quad$ has a continuous inverse $A^{-1}(s)$. It is clear that the operator conjugate to $A(s)$ is $A(s)$. One can show that the equation with the conjugate operator is properly solvable. From this it follows /14/ that Eq. (3.3) is solvable everywhere for Re $>0$. The operator $A(s)$ is an entire operator-valued function. The analyticity of $\tau(s)$ in the domain of analyticity of $\Phi$ for Res $\rightarrow 0$ follows from the continuity of $A^{-1}$.

We denote by $\tau_{1}$ and $\tau_{2}$ the solutions of Eq. (3.3), respectively, corresponding to the first and second terms in the expression for $\Phi$. The concluding estimates of the proof follow from inequalities (3.4):

$$
\begin{gathered}
\left\|\tau_{1}\right\|_{H_{1}} \leqslant x^{-1}\|K Q\|_{H_{1}},\left\|\tau_{2}\right\|_{H_{2}} \leqslant x^{-1} \mid \alpha s\|v\|_{L_{z}} \\
\left\|\tau_{2}\right\|_{L_{2}} \leqslant \zeta^{-1}(s)|\alpha s|\|v\|_{L_{z}}
\end{gathered}
$$

Using the results of Lema 3.1, Eq. (3.1) can be presented in the form of an operator equation in the space $H_{2}$ :

$$
\begin{aligned}
& B(s) \mathbf{v} \equiv B_{0}(s) \mathbf{v}-\alpha^{2} s K_{4}(s) \mathbf{v}=K_{3} \mathbf{F}+\mathrm{F}^{\prime} \\
& B_{0}(s) \mathbf{v} \equiv M(s) \mathbf{v}+[M(s)+\Lambda(s)] K_{2} \mathbf{v}+\rho s^{2} K_{3} v \\
& \left(K_{2}, \mathbf{v}, g\right)_{H_{z}}=\iiint \nabla \cdot \mathrm{v} \nabla \cdot \bar{g} g d \omega,\left(K_{3} \mathbf{v}, \mathrm{g}\right)_{H_{z}}=\iiint \mathrm{v} \cdot \vec{g} d \omega \\
& \left(K_{4} \mathbf{v}, \mathbf{g}\right)_{H_{2}}=\frac{1}{\alpha s} \iiint \tau_{2} \nabla \cdot \bar{g} d \omega, \quad\left(F^{\prime}, g\right)_{H_{4}}=\alpha \iiint \tau_{2} \Gamma \cdot \bar{s} d \omega
\end{aligned}
$$

The operator $K_{2}$ is continuous and positive, and takes $H_{2}$ into $H_{2}$. It is known $/ 15 /$ that $\left\|K_{2}\right\|=1$. The operator $K_{3}$ takes $H_{2}^{-1}$ continuously into $H_{2 *}$ The operator $K_{3}(s)$ takes $H_{2}$ continuously into $H_{2}$ and is completely continuous. The complete continuity of $K_{4}$ is a consequence of the inequality

$$
\left\|K_{4}(s) \vee\right\|_{H_{2}} \leqslant d_{1}\left(1+|s|^{2}\right)^{-1 / 3}\|v\|_{c_{1}}
$$

which is obtained from the estimate for $\tau_{2}$ in the space $L_{2}$ which was pointed out in Lemma 3.1 together with Sobolev's imbedding theorem. Here and below the $\vec{d}_{k}$ are arbitrary positive constants.

$B_{0}(s) \quad$ satisfies the equality

$$
\begin{gathered}
\left(B_{0}(s) \mathbf{v}, \mathbf{v}\right)_{K_{3}}=P(s)(\mathbf{v}, \mathbf{v})_{H_{2}}, P(s)=M(s)+k_{2}[\Lambda(s)+M(s)]+ \\
\rho k_{3} s^{2}, k_{2}=\left(K_{2} \mathbf{v}, \mathbf{v}\right)_{H_{3}}\|\mathbf{v}\|_{H_{3}}^{2} \equiv(0,1], k_{3}=\left(K_{3} \mathbf{v}, \mathbf{v}\right)_{H_{3}}\|\mathbf{v}\|_{k I_{n}} \in\left(0, k_{3}{ }^{\circ}\right)
\end{gathered}
$$

The stability requirement on the polynomial $P(s)$ for all $k_{2}$, $k_{3}$ in their domains of 
variation was put forward by /// as a sufficient condition for the solubility of Eq. (3.5) with $\alpha=0$. The solubility of Eq.(3.5) with $\alpha \neq 0$ is given by the following lemma.

Lemma 3.2. Suppose the polynomial $P(s)$ is stable, i.e. its roots have strictly negative real parts. Then a number $\alpha^{*}>0$ exists such that for $|\alpha|<\alpha^{*}$ Eq. (3.5) is uniquely soluble for lie $s \geqslant 0$ and the solution $v(s) \in E_{n}\left(0, H_{2}\right)$.

The proof is similar to the proof of Lemma 3.1 and is based on an a priori estimate. $B(s) \quad$ satisfies the equality

$$
(B(s) \mathbf{v}, \mathbf{v})_{H_{*}}=P(s)(\mathbf{v}, \mathbf{v})_{H_{\mathrm{z}}}-\alpha^{2} s\left(K_{\mathbf{4}}(s) \mathbf{v}, \mathbf{v}\right)_{H_{\mathrm{v}}}
$$

from which for Res $\geqslant 0$ there follows a necessary estimate for sufficiently small $a$ :

$$
\left|(B(s) \mathrm{v}, \mathrm{v})_{H_{3}}\right|>\left[d_{3}\left(1+|s|^{2 n}\right)^{1 / 2}-d_{3} \frac{a^{2}|\varepsilon|}{\left(1+|s|^{2}\right)^{1 / 2}}\right]\|\mathrm{v}\|_{H,}^{2},
$$

It is clear that the inequality obtained is satisfied for sufficiently small or large $|s|$ independently of $\alpha$.

In the case $n=1,2$ an a priori estimate can be obtained independently of $\alpha$. To do this the vector $\mathbf{g}$ in Eq. (3.1) is set equal to $s \mathbf{v}$, in (3.2) $\varphi=\tau$, and relation (3.1) is added to the complex conjugate of $(3.2)$, leading to the equality

$$
\left(\bar{s} B_{0}(s) \mathbf{v}, \mathbf{v}\right)_{H_{s}}+(A(s) \tau, \tau)_{H_{1}}=\left(\bar{s} K_{3} \mathbf{F}, \mathbf{v}\right)_{H_{s}}+(\overline{K Q, \tau})_{H_{1}}
$$

which, using previously obtained inequalities with Res $\geqslant 0$. leads to a necessary estimate on $v$ and $\tau$ independently of $\alpha$.

The solution estimates can be strengthened if one makes additional smoothness assumptions about the initial conditions. In particular, suppose $\mathbf{w}_{k}$ and $\theta_{0}$ are such that $f \in L_{2}(\omega)$ and $q \in L_{2}(\omega)$, then, using the results of Lemmas 3.1 and 3.2 , and properties of the operators $A(s) \quad$ and $B(s)$, one can show that

$$
\mathbf{v} \in E_{m}\left(0, L_{2}\right), \tau \in E_{1}\left(0, L_{2}\right), m=\max (2, n)
$$

The results obtained prove the existence and uniqueness of the generalized solution of problem B. Application of the theorem from $/ 7 /$ cited in Sect. 2 now guarantees the existence and uniqueness of a solution of problem $A$ in the following sense: for every pair of infinitely differentiable functions $\mathrm{g}(t) \in H_{2}$ and $\varphi(t) \in H_{1}$ which vanish when $t$ is larger than a certain number depending on $\mathrm{g}$ and $\varphi$, the solution $\mathbf{u}(t), \tau(t)$ satisfies the equation

$$
\begin{gathered}
\int_{0}^{\infty} \iiint\left(\nabla \mathbf{u} \cdot \cdot M\left(-\partial_{t}\right) \nabla_{\mathrm{g}}{ }^{T}+\nabla \cdot \mathbf{u}\left[M\left(-\partial_{t}\right)+\Lambda\left(-\partial_{t}\right)\right] \nabla \cdot g+\rho \mathbf{u} \cdot \partial_{t}{ }^{2} \mathrm{~g}-\right. \\
\left.\alpha \tau \nabla \cdot \mathrm{g}+x \nabla \tau \cdot \nabla \varphi-c \tau \partial_{t} \varphi-\alpha \mathbf{u} \cdot \nabla \partial_{t} \varphi-\mathbf{f} \cdot \mathrm{g}-q \varphi\right) d \omega d t
\end{gathered}
$$

We have thus proved the following theorem.

Theorem 3.1. Suppose $\mathrm{w}_{k} \in H_{2}, \theta_{0} \in H_{1}$, and the polynomial $p(s)$ is stable. Then there exists $\alpha^{*}>0$ (for $n=1,2, \alpha^{*}=\infty$ ) such that for $|\alpha|<\alpha^{*}$ problem $A$ has a unique generalized solution $u \in P_{n-*}\left(0, H_{3}\right)$ and $\tau \in P_{0}\left(0, H_{1}\right)$. If $w_{k}, \theta_{0}$ are such that $\mathbf{f} \in L_{2}$ and $q \in L_{2}$, then $\mathbf{u} \in P_{m}\left(0, L_{2}\right)$ and $\tau \in P_{1}\left(0, L_{2}\right)$ where $m=\max (2, n)$.

A sufficient condition for the local stability of a $\left(p, \theta^{\circ}\right)$-configuration follows directly from Theorem 3.1.

Theorem 3.2. Suppose that the conditions of Theorem (3.1) are satisfied. Then for $|\alpha|<\alpha^{*}$ the solution of the initial-boundary value problem $(1.6),(1.7),(1.9)-(1.11)$ is asymptotically stable in the following sense:

$$
\int_{M}^{\infty}\left\{\left\|\partial_{t}{ }^{k} \mathbf{w}\right\|_{H_{2}}^{2}+\left\|\partial_{t}{ }^{i} w\right\|_{L_{2}}+\|\theta\|_{H_{1}}^{2}+\left\|\partial_{t}^{j} \theta\right\|_{L_{3}}\right\} d t \rightarrow 0, \quad M \rightarrow \infty
$$

where $k=0, \ldots, n ; i=0, \ldots, m ; j=0,1$; and

$$
\left\|\partial_{t}{ }^{k-1} \mathbf{w}\right\|_{H_{1}} \rightarrow 0, \quad\left\|\partial_{t}{ }^{i-1} \mathbf{w}\right\|_{L_{1}} \rightarrow 0, \quad\|\theta\|_{L_{1}} \rightarrow 0, \quad t \rightarrow \infty
$$

uniformly in $t$.

The last assertion of Theorem 3.2 follows from the inequality /13/

$$
d_{t}\left\|\partial_{t}^{j} w\right\|_{H i}^{2} \leqslant \int_{M}^{\infty} \sum_{k=0}^{n}\left\|\partial_{t}^{k} w\right\|_{H_{z}}^{2} d t, \quad 0 \leqslant j<n
$$


For certain conditions on the set of initial data one can make more precise the manner in which $\theta(t)$ tends to zero. In particular, if $w_{k}, \theta_{0}$ are such that $q=0$, (for example, $\nabla \cdot \mathbf{w}_{k}=0$ and $\theta_{0}=0$ ), then with the conditions of Theorem 3.1 one can show that $\theta \in P_{m-1}\left(0, H_{1}\right) \cap P_{m}\left(0, L_{2}\right)$.

4. All the results presented above can be directly carried over to other initial-boundary value problems for compressible thermo-visco-elastic and incompressible visco-elastic bodics. Here we will merely note the necessary changes in the formulations of the theorems of sect. 3 .

In the case of a mixed boundary-value problem for a compressible thermo-visco-elastic body the space $H_{2}$ is replaced by $H_{3}$, where $H_{3}$ is the closure of vector functions continuously differentiable in $\omega$, satisfying homogeneous kinematic boundary conditions (w $\left.\right|_{\Sigma_{1}}=0$, $\left.\mathbf{N} \cdot \mathbf{w}\right|_{\mathbf{s}_{\mathbf{1}}}=0$ ), such that $\mathbf{w}$ satisfies Korn's inequality $/ 16 /$ in the norm induced by the scalar product

$$
(\mathbf{w}, \mathbf{u})_{H_{\mathrm{s}}}=\iiint \varepsilon(\mathbf{w}) \cdots \varepsilon(\mathrm{u}) d \omega
$$

The polynomial $P(s)$ is replaced by $P^{\prime}(s)=2 M(s)+\Lambda(s) k_{2}+\rho k_{3} s^{2}$. The parameter $k_{2} \in$ $\left(0,31\right.$ as a consequence of the inequality $\|\nabla \cdot w\|_{L_{2}} \leqslant \sqrt{3}\|\mathbf{w}\|_{H_{2}}$.

If the boundary conditions allow rigid-body displacements of the body, the assertions of Theorems 3.1 and 3.2 with the above changes hold only for the "deformation" part of the displacement orthogonal in $\boldsymbol{H}_{3}$ to the rigid displacement vectors.

In the same way one can investigate the case of more general thermal boundary conditions.

The initial-boundary value problem for an incompressible visco-elastic body is treated similarly. Here the spaces $H_{2}$ and $H_{3}$ are obtained by the closure in corresponding norms of solenoidal vector functions, $i . e$. those vector functions satisfying the condition $\nabla \cdot w=0$. For the first boundary-value problem $P(s)=M(s)+\rho k_{3} s^{2}$, and for the others $P^{\prime}(s)=2 M(s)+$ $\rho k_{3} s^{2}$.

Analysis of the properties of the polynomials $P(s)$ and $P^{\prime}(s)$ shows that a necessary. and for $n=1,2$ also a sufficient, condition for the asymptotic stability of a (p, $\left.\theta^{\circ}\right)$ configuration is that the inequalities $\mu_{k}>0, \lambda_{k}+2 \mu_{k}>0(k=0, \ldots, n)$ for the first boundary-value problem and $\mu_{k}>0,3 \lambda_{k}+2 \mu_{k}>0$ for all boundary-value problems with $l \in(0,11$. In the case of an incompressible body these boundary conditions are the inequalities $\mu_{k}>0$. These inequalities impose definite restrictions on relations (1.7) and (1.8) defining the response of the material to small deformations near the HSS.

The restrictions imposed by the conditions of Theorem 3.1 for $n>2$ on $\alpha$ are, in general, necessary.

As an example we will consider the problem of the stability of a rectangle with sides $a, b$, in contact over its entire surface with a smooth rigid surface and with Neumann conditions on the temperature $\left(\Sigma=\Sigma_{\mathbf{s}}=\Sigma_{\mathrm{b}}\right)$. A solution $\mathbf{w}=u \mathrm{i}_{\mathbf{1}}+\boldsymbol{v} \mathbf{l}_{\mathbf{3}}, \boldsymbol{\theta}$ satisfying the boundary conditions is sought in the form

$$
\begin{gathered}
u(x, y, t)=\sum_{n, m=0}^{\infty} u_{n m} e^{v_{n m} t} \sin \frac{\pi n}{a} x \cos \frac{\pi m}{b} y, \quad v(x, y, t)= \\
\sum_{n, m=0}^{\infty} v_{n m} e^{v_{n m} t} \cos \frac{\pi n}{a} x \sin \frac{\pi m}{b} y, \quad \theta(x, y, t)= \\
\sum_{n, m=0}^{\infty} \theta_{n m} e^{v_{m n} t} \cos \frac{\pi n}{a} x \cos \frac{\pi m}{b} y
\end{gathered}
$$

Substituting this into Eqs.(1.6), (1.9) we obtain a linear homogeneous system of algebraic equations in $u_{n m}, v_{n, n}$ and $\theta_{n m}$. The condition for its non-trivial solubility can be shown to be the vanishing of the expression

$$
\begin{gathered}
D(v, \beta, \alpha)=\left[\rho v^{2}+\beta M(v)\right]\left\{\alpha^{2} \beta v+(c v+x \beta)\left[\rho v^{2}+\beta(\Lambda(v)+2 M(v))\right]\right\} \\
v=v_{n m}, \quad \beta=(\pi n / a)^{2}+(\pi m / b)^{2}
\end{gathered}
$$

If the equation $D(v, \beta, \alpha)=0$ has a root $v_{k}$ with positive real part, this means that the problem has an exponentially increasing solution and therefore the $\left(p, \theta^{\circ}\right)$-configuration is Lyapunov-unstable for the rectangle. It is clear that for $\alpha=0$ the stability requirement for all $\beta$ is equivalent to the stability requirement of the polynomial $P(v)$ in the conditions of Theorem 3.1 .

One can show that for $n=1,2$ a necessary and sufficient condition for the stability of $D(\nu, \beta, \alpha)$ for all $\beta$ and $\alpha$ is the satisfaction of the inequalities $\lambda_{k}+2 \mu_{k}>0, \mu_{k}>0(k=0$, $\ldots, 2)$ Analysis of the stability of the polynomial $D(v, \beta, \alpha)$ for $n=3$ shows that for some $\alpha^{*}(\beta)$ the Hurwitz stability criteria for $D(v, \beta, \alpha)$ are violated. via the parameter $\beta$ the critical parameter $\alpha^{*}$ also depends on the dimensions of the 
domain. One can show that $\alpha^{*}=O(\beta)$ for $\beta \rightarrow \infty$ and $\alpha^{*}=0\left(\beta^{-1 / 1}\right)$ for $\beta \rightarrow 0$. Unlike the conditions on $\lambda_{k}$ and $\mu_{k}$, the restrictions on $\alpha$ for $n>2$ cannot be considered as a restriction on the defining relations for thermo-visco-elastıc bodies ln the case of small deformations (1.7) because the constant $\alpha^{*}$ also depends on the domain occupied by the body.

The authors thank L.M. Zubov and L.P. Lebedev for their help with this paper.

\section{REFERENCES}

1. LUR'YE A.I., The Theory of Elasticity, Nauka, Moscow, 1970.

2. ZUBOV L.M., On local uniqueness conditions for a hydrostatic compression state of an elastic body, Prikl. Mat. Mekh., 44, 3, 1970.

3. ARUTYUNYAN N.KH. and DROZDOV A.D., A stability theory for visco-elastic bodies under finite deformations, Dokl. Akad. Nauk SSSR, 276, 5, 1984.

4. ARUTYUNYAN N.KH. and DROZDOV A.D., The stability of inhomogeneous ageing visco-elastic bodies, Voprosy Sudostroyeniya, Ser. Proektirovanie Sudov, TsNII "Rumb", Leningrad,42, 1985.

5. DROZDOV A.D. and SOLOMENTSEV Yu.E., On the stability of non-linear visco-elastic bodies, IzV. Akad. Nauk SSSR, MTT, 6, 1986.

6. AGRANOVICH M.S. and VISHIK M.I., Elliptical problems with a parameter and parabolic problems of general form, Usp. Mat. Nauk, 19, 3, 1964.

7. LEBEDEV L.P., On the stability of naturally unstressed states of visco-elastic bodies, Prikl. Mat. Mekh., 39, 6, 1975.

8. TRUESDELL K., A First Course on the Rational Mechanics of Continuous Media, Mir, Moscow, 1975.

9. LUR'YE A.I., Non-linear Elasticity Theory, Nauka, Moscow, 1980.

10. SEDOV L.I., The Mechanics of Continuous Media, 1, Nauka, Moscow, 1983.

11. IL'YUSHIN A.A. and POBEDRYA B.E., Foundations of the Mathematical Theory of Thermo-ViscoElasticity, Nauka, Moscow, 1970.

12. PETROV N. and BRANKOV I., Modern Problems in Thermodynamics, Mir, Moscow, 1986.

13. LYONS J.-L. and MAGENES E., Inhomogeneous Boundary-value Problems and their Applications, Mir, Moscow, 1971.

14. KREIN S.G., Linear Equations in Banach Spaces, Nauka, Moscow, 1971.

15. MIKHLIN M.G., Spectrum of a bundle of operators from elasticity theory, Usp. Mat. Nauk, $28,3,1973$.

16. MOSOLOV P.P. and MYASNIKOV V.P., A proof of Korn's inequality, Dokl. Akad. Nauk SSSR, 201, 1, 1971 . 